\title{
Probing the quantum ground state of a spin-1 Bose-Einstein condensate with cavity transmission spectra
}

\author{
J. M. Zhang, ${ }^{1,2}$ S. Cui, ${ }^{2}$ H. Jing, ${ }^{2}$ D. L. Zhou, ${ }^{1}$ and W. M. Liu ${ }^{1}$ \\ ${ }^{1}$ Beijing National Laboratory for Condensed Matter Physics, \\ Institute of Physics, Chinese Academy of Sciences, Beijing 100080, China \\ ${ }^{2}$ Department of Physics, Henan Normal University, Xinxiang 45300\%, China
}

\begin{abstract}
We propose to probe the quantum ground state of a spin-1 Bose-Einstein condensate with the transmission spectra of an optical cavity. By choosing a circularly polarized cavity mode with an appropriate frequency, we can realize coupling between the cavity mode and the magnetization of the condensate. The cavity transmission spectra then contain information of the magnetization statistics of the condensate and thus can be used to distinguish the ferromagnetic and antiferromagnetic quantum ground states. This technique may also be useful for continuous observation of the spin dynamics of a spinor Bose-Einstein condensate.

PACS numbers: 03.75.Mn, 32.10.Dk, 37.10.Vz, 42.50.Pq
\end{abstract}

Spinor Bose-Einstein condensates (BECs) distinguish themselves from their scalar counterparts by their much richer internal structures and dynamics [1, 2]. Based upon the single mode approximation (SMA), which is valid in most cases, the quantum eigenstates of a spinor BEC has been extensively and thoroughly studied with sophisticated algebraic techniques [3, 4, 5]. Especially, for the spin- 1 case, which we focus on in this paper, it is found that there are two possibilities for the ground state. Depending on a single parameter, the ground state can be either of an antiferromagnetic (AFM) or a ferromagnetic (FM) nature. The two phases differ in their rotational properties - the AFM ground state is a $S U(2)$ singlet and thus isotropic [6]; while the FM ground state is a $S U(2)$ multiplet heavily degenerate and thus can be well directed (up to the uncertainty principle associated with the $S U(2)$ algebra). From the point of view of quantum phase transition, these two phases deserve to be distinguished experimentally for their own sake.

In this paper we propose a method based on cavity quantum electrodynamics techniques to fulfill this object. The basic idea is similar to that of Mekhov et al. in Ref. [7], where they proposed to probe the superfluidity-Mott insulator transition of cold atoms in optical lattices with cavity transmission spectra. In the dispersive regime, atoms inside the cavity shift the cavity frequency and in turn influence the cavity transmission spectra. The superfluidity and Mott-insulator phases differ in their atom number statistics and thus lead to drastically different cavity transmission spectra, which can be used inversely to differentiate the two phases. In their proposal, it is adequate to treat the atoms in a two-level fashion with the atomic levels structureless. However, here in our case, we have to go beyond the two-level model and take into account the detailed and realistic atomic level structure. In this way, we can retrieve the vector polarizability 8 of the atom that is dropped artificially in the two-level model. It follows that by choosing a circularly polarized cavity mode, the cavity photon couples not only to the total atom number but also to the total magnetization.
The latter effect allows us to do our job, since the cavity transmission spectra carry information of the magnetization statistics of the BEC, which are absolutely different in the two phases.

We assume a spin-1 BEC of $N$ alkali atoms of a certain type is loaded inside an optical cavity [9]. This system is probed with a $\sigma_{+}$polarized laser so that the relevant cavity mode coupled with the atoms is also $\sigma_{+}$polarized. The bare frequency $\omega_{c}$ of this cavity mode is chosen to resolve the alkali $D$ line (fine structure) and placed relatively near the $D_{1}$ transition [10]. However, the detuning between the $D_{1}$ transition and the cavity mode frequency is still large enough not to resolve the hyperfine structures of the $D_{1}$ line. More precisely, we have $\Delta_{H F S 1} \ll\left|\omega_{c}-\omega_{D 1}\right| \ll\left|\omega_{c}-\omega_{D 2}\right|$, where $\Delta_{H F S 1}$ is the magnitude of the hyperfine splitting of the $D_{1}$ line, $\omega_{D 1}$ and $\omega_{D 2}$ are the average frequency of the hyperfine levels of $D_{1}$ and $D_{2}$, respectively. In the appendix we show that the effective Hamiltonian describing the atom-cavity photon interaction is ( $\hbar=1$ throughout in this paper)

$$
H_{\text {int }}=\sum_{m}\left(1+\frac{m}{2}\right) U_{0} \int d x u^{2}(x) \psi_{m}^{\dagger}(x) \psi_{m}(x) a^{\dagger} a .
$$

Here $\psi_{m}(x)(m=0, \pm 1)$ is the field annihilation operator for an atom in the hyperfine state $|F=1, m\rangle$ at position $x$ (the quantization direction is along the cavity axis and is denoted as the $z$-direction), and $a$ is the annihilation operator for the cavity photon. The cavity mode function with its maximum normalized to unity is denoted as $u(x)$. The parameter $U_{0}$ characterizes the strength of the ac-Stark shifts of the atomic levels per photon, in the viewpoint of the atoms; or alternatively, the magnitude of the cavity frequency shift per atom, in the viewpoint of the photons. Formally, we can write $U_{0}$ as $U_{0}=g_{0}^{2} /\left(\omega_{c}-\omega_{D 1}\right)$ by introducing an effective atomphoton coupling strength $g_{0}$, which incorporates the overall effect of coupling with all the hyperfine levels in the $D_{1}$ line.

Here some comments are in order. The main feature of Hamiltonian (1) is the spin dependence of the level shifts, 
which can be interpreted in terms of a fictitious magnetic field. In most experiments aiming to study intrinsic spin dynamics of a spinor BEC, this effect is (and should be) avoided or minimized by taking the laser beam to be linearly polarized or with a detuning too large to resolve the $D$ line or both (an exception being Ref. [11]). However, for the purpose of continuous observation of spin dynamics and spin magnetization, this effect proves to be useful. Actually, it is exactly the same physics that underlies the Faraday rotation spectroscopy developed recently for in situ observation of a spinor BEC [12, 13, 14]. For if the cavity mode is $\sigma_{-}$polarized, the plus sign in front of $\frac{m}{2}$ in Eq. (1) should be replaced with a minus sign. That is, the $\sigma_{+}$and $\sigma_{-}$polarized light respond differently to a given atomic sample - the essence of the Faraday rotation effect.

For the spinor BEC, we assume the SMA is valid and rewrite the atomic field operators as $\psi_{m}(x)=\phi(x) c_{m}$. Here $\phi(x)$ denotes the common spatial mode (normalized to unity) for the three spin components and $c_{m}$ is the annihilation operator associated with the corresponding spin component. Under the SMA, we can rewrite Hamiltonian (11) as

$$
H_{i n t}=U\left(\hat{N}+\frac{1}{2} \hat{M}\right) a^{\dagger} a,
$$

where $\hat{N}=\sum_{m} c_{m}^{\dagger} c_{m}$ is the total atom number and $\hat{M}=\sum_{m} m c_{m}^{\dagger} c_{m}$ is the total magnetization. The parameter $U \equiv U_{0} \int d x u^{2}(x) \phi^{2}(x)$ is proportional to the overlap between the cavity mode function and the condensate mode function. In general, the extension of a condensate is much larger than the period of $u^{2}(x)$ in the axial direction while much smaller than its waist in the transverse direction. Therefore, for a condensate placed around the axis of the cavity, the integral can be well approximated with $1 / 2$, i.e., $U \simeq U_{0} / 2$. We note that $\hat{N}$ and $\hat{M}$ commute with both $H_{\text {int }}$ and the Hamiltonian of the spinor BEC itself. Therefore, we have a quantum non-demolition measurement.

Assume the probe laser is of frequency $\omega_{p}$. The Heisenberg equation (in the frame rotating at $\omega_{p}$ ) for the cavity mode annihilation operator is

$$
\dot{a}=-i\left(\omega_{c}-\omega_{p}+U\left(\hat{N}+\frac{1}{2} \hat{M}\right)\right) a-\kappa a+\eta,
$$

where $\kappa$ is the damping rate of the cavity mode and $\eta$ the driving amplitude. The damping term leads to the stationary solutions of $a$ and the photon number [7],

$$
\begin{gathered}
a_{s t}=\frac{\eta}{\kappa+i(\Delta+U \hat{M} / 2)}, \\
\hat{n}_{s t}=a_{s t}^{\dagger} a_{s t}=\frac{\eta^{2}}{\kappa^{2}+(\Delta+U \hat{M} / 2)^{2}},
\end{gathered}
$$

where $\Delta=\omega_{c}+U N-\omega_{p}$ is the shifted cavity-probe detuning. The expectation value of $\hat{n}_{s t}$ over a prescribed state of the spinor BEC is then

$$
n_{s t}=\left\langle\hat{n}_{s t}\right\rangle=\sum_{M=-N}^{N} f_{M} \frac{\eta^{2}}{\kappa^{2}+(\Delta+U M / 2)^{2}},
$$

where $f_{M}$ is the probability for $\hat{M}$ taking value $M$ in the prescribed state. We have $\sum_{M} f_{M}=1$, where the sum is over all the possible values of $M$. As pointed out in Ref. [15], the total photon number contains incoherent contributions of a series of coherent states, the $M$-th of which is of amplitude $\eta /(\kappa+i(\Delta+U M / 2))$ and of weight $f_{M}$. Mathematically, we see from Eq. (5) that $n_{s t}$, as a function of the detuning $\Delta$, is a weighted sum of a series of Lorentz functions, which are evenly displaced with $U / 2$ and of full-width half-maximum $2 \kappa$. The point is that the cavity transmission spectra (proportional to $n_{s t}$ ) contain information of the atomic statistics embodied in $f_{M}$.

We now apply the formalism developed above to the two possible phases of a spin-1 BEC. For a spin-1 BEC in the SMA, the Hamiltonian accounting for the spin dynamics is $H_{s}=W \mathbf{S}^{2}[3]$, with the spin operator $\mathbf{S} \equiv\left(S_{x}, S_{y}, S_{z}\right)=c_{m}^{\dagger}\left(S_{x}^{m n}, S_{y}^{m n}, S_{z}^{m n}\right) c_{n}$. The parameter $W$ is proportional to the difference of the s-wave scattering lengthes in the two total spin channels $(F=0$ or 2). Obviously, the sign of $W$ determines the nature of the ground state. If $W>0$, the ground state is of total spin $S=0$ and given explicitly as [4, 5,6$]$

$$
|G\rangle_{A F M}=|S=0, M=0\rangle \propto\left(A^{\dagger}\right)^{N / 2}|0\rangle,
$$

where the scalar (rotationally invariant) operator $A^{\dagger} \equiv$ $c_{0}^{\dagger 2}-2 c_{+1}^{\dagger} c_{-1}^{\dagger}$. This state is a singlet and also the unique ground state. For this AFM ground state, the magnetization has no fluctuations at all. The only nonvanishing term in Eq. (5) is $M=0$. Therefore, by scanning the probe frequency $\omega_{p}$, we get the intracavity photon number as a single Lorentz function,

$$
n_{s t}(\Delta)=\frac{\eta^{2}}{\kappa^{2}+\Delta^{2}}
$$

with a width of $2 \kappa$ and maximum height of unity (in units of $\eta^{2} / \kappa^{2}$, the maximum possible intracavity photon number). Note that it is of the same shape as that of a bare cavity, though the center is shifted by $U N$.

If $W<0$, the ground state is of total spin $S=N$ and $2 N+1$ fold degenerate. Explicitly, we have as a set of orthonormal basis [4, 5],

$$
|G\rangle_{F M}=|S=N, M\rangle \propto\left(S_{-}\right)^{N-M}\left(c_{+1}^{\dagger}\right)^{N}|0\rangle,
$$

where $S_{-}=\sqrt{2}\left(c_{0}^{\dagger} c_{+1}+c_{-1}^{\dagger} c_{0}\right)$ is the lowering operator for $S_{z}$. These $2 N+1$ states each have a definite magnetization, thus the corresponding transmission spectra is also a single Lorentz function (but the center of which may vary). However, here we are most interested in the experimentally most accessible states - angular momen- 
tum coherent states - states that can be obtained by rotating the $z$-aligned state $|N, N\rangle$,

$$
e^{-i \theta \vec{\zeta} \cdot \vec{S}}|N, N\rangle=\frac{\left(x_{+1} c_{+1}^{\dagger}+x_{0} c_{0}^{\dagger}+x_{-1} c_{-1}^{\dagger}\right)^{N}}{\sqrt{N !}}|0\rangle .
$$

Here $\vec{\zeta}=(-\sin \phi, \cos \phi, 0)$ perpendicular to the $z$-axis is the axis of rotation, while $\theta$ is the angle of rotation or inclination angle. The $x_{i}$ 's are determined by $e^{-i \theta \vec{\zeta} \cdot \vec{S}} c_{+1}^{\dagger} e^{i \theta \vec{\zeta} \cdot \vec{S}}=x_{+1} c_{+1}^{\dagger}+x_{0} c_{0}^{\dagger}+x_{-1} c_{-1}^{\dagger}$. The state constructed in Eq. (91) defines a direction $(\theta, \phi)$ in that it is the eigenstate of $S_{n(\theta, \phi)}=\sin \theta \cos \phi S_{x}+\sin \theta \sin \phi S_{y}+$ $\cos \theta S_{z}$ with eigenvalue $N$. These states can be readily prepared with a proper radio-frequency (rf) pulse from the state $|N, N\rangle[16]$. For such a state, the weight coefficients of the Fock states $\left|N_{+1}, N_{0}, N_{-1}\right\rangle$ follow the triplenominal distribution

$$
P\left(N_{+1}, N_{0}, N_{-1}\right)=\frac{N !}{N_{+1} ! N_{0} ! N_{-1} !} p_{+1}^{N_{+1}} p_{0}^{N_{0}} p_{-1}^{N_{-1}},
$$

with $p_{i}=\left|x_{i}\right|^{2}$ and $\sum_{i} p_{i}=1$. Explicitly, we have $\left(p_{+1}, p_{0}, p_{-1}\right)=\left(\cos ^{4} \frac{\theta}{2}, 2 \cos ^{2} \frac{\theta}{2} \sin ^{2} \frac{\theta}{2}, \sin ^{4} \frac{\theta}{2}\right)$. Note that $p_{i}$ are independent of the azimuth angle $\phi$. This is reasonable since all angular momentum coherent states with the same inclination angle $\theta$ are connected by rotations around the $z$-axis. Therefore they share the same distribution $P\left(N_{+1}, N_{0}, N_{-1}\right)$.

The probability distribution function $f_{M}$ can be generated in a random walk process. Image a particle, initially at the origin, performing random walks on the real axis. At each step, the particle either moves rightward or leftward or just keeps still, with probabilities $p_{+1}, p_{-1}$, and $p_{0}$, respectively. Then $f_{M}$ is just the probability of arriving at $M$ after $N$ steps. In other words, $M$ is the sum of $N$ independent random variables $\left\{X_{k}, 1 \leq k \leq N\right\}$, the common distribution function of which is $\operatorname{Pr}\left(X_{k}=i\right)=p_{i}$, with $i=0, \pm 1$. This interpretation enables us to calculate $f_{M}$ both analytically and numerically. Analytically, for $N \gg 1$, by the central limit theorem, we can approximate $f_{M}$ with the normal distribution

$$
f_{M}=\frac{1}{\sqrt{2 \pi} \sigma} \exp \left(-\frac{\left(M-M_{c}\right)^{2}}{2 \sigma^{2}}\right)
$$

where $M_{c}=N \cos \theta$ and $\sigma=\sqrt{N} \sigma_{0}$ (here $\sigma_{0}=\sqrt{\frac{1}{2} \sin ^{2} \theta}$ being the variance of $X_{k}$ ) are respectively the average value and variance of $M$. Numerically, the characteristic function of $f_{M}$ is $\chi(t)=\left(p_{+1} e^{+i t}+p_{0}+p_{-1} e^{-i t}\right)^{N}$. By an inverse Fourier transform, we get $f_{M}$ determined (it is just the coefficient of $e^{i M t}$ in the expansion of $\chi(t)$ ). The two approaches yield the same result up to a high precision. In Fig. 1(a), we show the distribution $f_{M}$ for four different inclination angles with the total atom number $N=10^{6}$. We see the larger the absolute value of $\sin \theta$, the larger the fluctuations of $M$. For all cases, we see a clear $\sqrt{N}$ scaling of $\sigma$.
We calculate the intracavity photon number $n_{s t}$ by approximating $f_{M}$ with Eq. (11) and replacing the sum with an integral. The integral can be further understood as the convolution of the probability distribution function $f_{M}$ and a Lorentz function. We can then use the convolution theorem to first solve the Fourier transforms of the two functions respectively, form their product, and then take an inverse Fourier transform. We get

$$
n_{s t}(\Delta)=\frac{\eta^{2}}{2 \kappa} \int_{-\infty}^{+\infty} d t e^{i t\left(\Delta-\Delta_{c}\right)} \times e^{-\kappa|t|-t^{2} U^{2} \sigma^{2} / 8},
$$

where $\Delta_{c}=-M_{c} U / 2$. Obviously, the procedure can be done inversely, i.e., from the cavity transmission spectra observed experimentally, we can infer the distribution of the magnetization by Fourier transforms.

We can read two facts from Eq. (12). Firstly, the shape of $n_{s t}$ as a function of $\Delta$ is shifted by $\Delta_{c}$ from that depicted in Eq. (7). This overall shift is due to the average deviation of $M$ from zero and thus has a classical nature. Secondly, the shape of $n_{s t}$ is broadened compared to that in Eq. (7), which is due to the fluctuations of $M$ around the mean and thus has a quantum nature. The shift and broadening are appreciable if $\left(M_{c} U, \sigma U\right) \gg \kappa$, or in terms of $N,(N U, \sqrt{N} U) \gg \kappa$. Currently, $g_{0}$ is about $2 \pi \times 15 \mathrm{MHz}$ and the line width $\kappa$ of the cavity can be made as small as $2 \pi \times 1.0 \mathrm{MHz}[17,18,19]$. By taking the detuning $\Delta_{c a}=\omega_{c}-\omega_{D 1}$ to $2 \pi \times 40 \mathrm{GHz}$, we get $U \sim 2 \pi \times 2.8 \mathrm{kHz}$. The atom number of a spinor BEC is typically on the order of $10^{6}$. Thus we see the first condition $N U \gg \kappa$ is well satisfied while the second condition $\sqrt{N} U \gg \kappa$ is marginally satisfied. In the near future, we expect $\kappa$ can be reduced by one order and then the second condition can be well met also.

In Fig. 1(b), we present the intracavity photon number at steady state $n_{s t}$ as a function of the detuning $\Delta$. For the AFM ground state, the curve (solid line) is a simple Lorentzian centered at $\Delta_{c}=0$ and with width $2 \kappa$. This is the only possibility. However, for the FM ground states, depending on the inclination angle $\theta$, the center and width of the curve vary widely (the figure may be a bit misleading, but note that we have artificially shifted the curves to a common center; see the labels of the horizontal axes). Especially, the broadening effect is apparent compared to the AFM case (here we have $\sqrt{N} U / \kappa=10)$. Thus the AFM and FM ground states can be distinguished on one hand by the different shapes of the corresponding spectra [20] and on the other hand by their responses to rf pulses (or a transverse magnetic field). Under the action of rf pulses, the AFM ground state remains unaltered since it is rotationally invariant, so the cavity transmission spectra are unchanged. In contrast, a FM ground state can be tickled to other directions and thus both the center and the width of the transmission spectra will change.

An alternative and more convenient method may be just reversing the polarization of the probe laser. Because the $\sigma_{+}$and $\sigma_{-}$polarized cavity modes couple respectively to $\hat{M}$ and $-\hat{M}$, it is easy to see that for the 

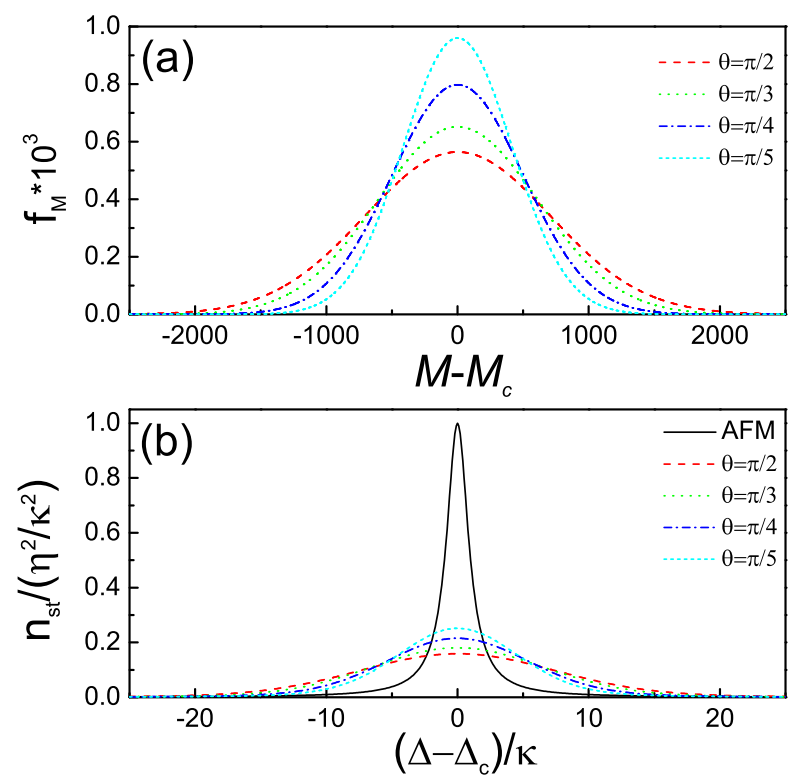

FIG. 1: (Color online) (a) Probability distribution function $f_{M}$ of the magnetization $M$ of ferromagnetic (FM) ground states with different inclination angles. (b) Intracavity photon number at steady state $n_{s t}$ as a function of the detuning $\Delta$. The solid line corresponds to the antiferromagnetic (AFM) ground state. Other lines correspond to FM ground states in (a). The parameters are $N=10^{6}$ and $U=0.01 \kappa$. For the horizontal labels, $M_{c}=N \cos \theta$ and $\Delta_{c}=-M_{c} U / 2$ in the FM case, while $M_{c}=0$ and $\Delta_{c}=0$ in the AFM case.

AFM case, the transmission spectra are unaffected; while for the FM case, the transmission spectra are reflected to be centered at $-\Delta_{c}$. The distance between the two centers is on the order of $N U$, which is much larger than $\kappa$, therefore this effect can be readily observed. This method makes evident the similarity between our approach and the Faraday rotation spectroscopy.

In summary, we have shown that by choosing a circularly polarized cavity mode, we can achieve coupling between the photon number with the magnetization, i.e. the $z$-component of the total spin of the spinor BEC. Full statistics of the magnetization of the spinor BEC can be extracted from the cavity transmission spectra and this allows us to discern the AFM and FM phases, which due to their different rotational properties, possess absolutely different magnetization statistics. Our discussion has been focused on the thermodynamical properties of the BEC (ground state oriented). However, it is clear that the technique can also be used for observing the real-time evolution of a spinor BEC. Moreover, since the condition $N U \gg \kappa$ can be readily fulfilled, the sensitivity can be high.

It should be emphasized that our discussion has been greatly simplified by neglecting the center-of-mass motion of the BEC and by the SMA [without the two approximations, we cannot reduce Eq. (11) to Eq. (2)]. These approximations are reasonable if the probe is weak enough. For future works, however, the strong probe case is worth investigation. If the probe is so strong that the intracavity optical lattice cannot be neglected, center-ofmass motion of the spinor BEC can be excited. Moreover, since the optical lattice felt by different spin components are different, a dynamical SMA is inappropriate. The situation is further complicated by the back-action of the BEC on the cavity mode. In the end, this system may exhibit many interesting nonlinear effects [21].

This work was supported by NSF of China under Grant Nos. 10874235, 60525417, and 10775176, NKBRSF of China under Grant Nos. 2009CB930704, 2006CB921400, 2006CB921206, and 2006AA06Z104. J. M. Z. would like to thank J. Ye for stimulating discussions.

\section{APPENDIX: EFFECTIVE HAMILTONIAN}

In this appendix we review the derivation of the effective Hamiltonian (11). The main concern is to demonstrate the Zeeman-like effect. General theory about this effect has actually been well established [22, 23, 24]. We include the derivation here just to serve our concrete purpose. For simplicity, we take the optical field to be classical, generalization to the quantum case is straightforward. The alkali atom in question is assumed to be in the $n^{2} S_{1 / 2} F=1$ hyperfine ground state manifold. A laser with electric field $\vec{E}(t)=\vec{E} \exp \left(-i \omega_{f} t\right)+$ c.c. couples the atom to the $n^{2} P_{1 / 2}$ excited state manifold $\left(D_{1}\right.$ line). Here it is assumed that the frequency of the laser is chosen so that the fine structure of the alkali $D$ line is well resolved while the hyperfine structure is not. This is feasible since the fine structure splitting is generally three or four orders larger in magnitude than the hyperfine structure splitting for the alkali $D$ line. For instance, the fine splitting of the $D$ line of ${ }^{87} \mathrm{Rb}$ is $2 \pi \times 7200 \mathrm{GHz}$, while the hyperfine splitting of the $D_{1}$ line is $2 \pi \times 814.5$ $\mathrm{MHz}$.

We aim to derive an effective Hamiltonian confined in the $F=1$ ground state subspace. By second order perturbation theory and taking the rotating wave approximation, we have [25]

$$
H_{e f f}=\frac{P_{g ; F=1}\left(\vec{E}^{*} \cdot \vec{d}\right) P_{D 1}(\vec{d} \cdot \vec{E}) P_{g ; F=1}}{\omega_{f}-\omega_{D 1}},
$$

where $\vec{d}$ is the electric dipole operator and $P_{g ; F=1}$ and $P_{D 1}$ are the projection operators on the $F=$ 1 hyperfine ground state manifold and $D 1$ fine excited state manifold respectively. That is, $P_{g ; F=1}=$ $\sum_{m}\left|n^{2} S_{1 / 2} ; F=1, m\right\rangle\left\langle n^{2} S_{1 / 2} ; F=1, m\right|$ and $P_{D 1}=$ $\sum_{F, m}\left|n^{2} P_{1 / 2} ; F, m\right\rangle\left\langle n^{2} P_{1 / 2} ; F, m\right|$, where the sum is over all the possible values of $F$ and $m$. For the latter, we can also transfer from the coupled representation to the uncoupled representation, i.e., equivalently we have

$$
P_{D 1}=P_{e ; J=1 / 2} \otimes P_{I}
$$


Here $P_{e ; J=1 / 2}$ is purely an electronic projection operator onto the $J=1 / 2$ excited state subspace while $P_{I}$ is purely a nuclear projection operator. We can also define the project operator

$$
P_{g}=P_{g ; J=1 / 2} \otimes P_{I}=P_{g ; F=1}+P_{g ; F=2},
$$

which projects onto the ground state manifold up to the fine structure. Substituting (A.2) and A.3 into A.1 and noting that $P_{g} P_{g ; F=1}=P_{g ; F=1}$, we rewrite the effective Hamiltonian as

$$
\begin{aligned}
H_{e f f} & =P_{g ; F=1} \frac{P_{g}\left(\vec{E}^{*} \cdot \vec{d}\right) P_{D 1}(\vec{d} \cdot \vec{E}) P_{g}}{\omega_{f}-\omega_{D 1}} P_{g ; F=1} \\
& =P_{g ; F=1}\left(\frac{P_{g ; J=1 / 2}\left(\vec{E}^{*} \cdot \vec{d}\right) P_{e ; J=1 / 2}(\vec{d} \cdot \vec{E}) P_{g ; J=1 / 2}}{\omega_{f}-\omega_{D 1}} \otimes P_{I}\right) P_{g ; F=1}
\end{aligned}
$$

Note that in the parentheses we have successfully separated the electronic part from the nuclear part. This is important since the external electric field couples with the electrons but not the nucleus. We define $\vec{D}=$ $P_{e ; J=1 / 2} \overrightarrow{d P} P_{g ; J=1 / 2}$, which is a vector operator (and so is $D^{\dagger}$ ). The electronic part in the parentheses in (A.4) is then in the form $\left(\vec{E}^{*} \cdot \vec{D}^{\dagger}\right)(\vec{D} \cdot \vec{E})$, which can be rewritten in the form $D_{i}^{\dagger} D_{j} E_{i}^{*} E_{j}$, the scalar contraction of two rank-2 tensors, with the atomic and optical parts separated. We can decompose the dyadic $D_{i}^{\dagger} D_{j}$ into rank-0, 1, 2 irreducible tensors,

$$
D_{i}^{\dagger} D_{j}=\frac{1}{3}\left(\vec{D}^{\dagger} \cdot \vec{D}\right) \delta_{i j}+\frac{1}{2}\left(D_{i}^{\dagger} D_{j}-D_{j}^{\dagger} D_{i}\right)+\left[\frac{1}{2}\left(D_{i}^{\dagger} D_{j}+D_{j}^{\dagger} D_{i}\right)-\frac{1}{3}\left(\vec{D}^{\dagger} \cdot \vec{D}\right) \delta_{i j}\right],
$$

with $E_{i}^{*} E_{j}$ done similarly. We note that since $D^{\dagger} D$ acts on a two-dimensional Hilbert space, the rank-2 irreducible part vanishes identically. We can then contract the corresponding irreducible tensors to form scalars. The result is

$$
\frac{1}{3}\left(\vec{D}^{\dagger} \cdot \vec{D}\right)\left(\vec{E}^{*} \cdot \vec{E}\right)+\frac{1}{2}\left(\vec{D}^{\dagger} \times \vec{D}\right) \cdot\left(\vec{E}^{*} \times \vec{E}\right)
$$

Using the Wigner-Eckart theorem, we see that in the $J=1 / 2$ ground state subspace the scalar operator $\vec{D}^{\dagger} \cdot \vec{D}$ is proportional to the identity operator while the vector operator $\vec{D}^{\dagger} \times \vec{D}$ to $\vec{J}$. Thus the above equation is equivalent to

$$
|\vec{E}|^{2}\left(\alpha-i \beta\left(\vec{e}^{*} \times \vec{e}\right) \cdot \vec{J}\right)
$$

with $\alpha$ and $\beta$ being some constants and $\vec{e}=\vec{E} /|\vec{E}|$ being the laser polarization vector. Substituting this result into
(A.4) and using again the Wigner-Eckart theorem, we have finally

$$
H_{e f f}=\frac{|\vec{E}|^{2}}{\omega_{f}-\omega_{D 1}}\left(\alpha-i \gamma\left(\vec{e}^{*} \times \vec{e}\right) \cdot \vec{F}\right)
$$

with $\gamma$ being some constant incorporating the Landé factor 26]. The first term describes a center-of-mass shift of the ground state multiplet while the second term is in effect a Zeeman term - the very effect we are seeking. Note that for a linearly polarized laser the second term vanishes identically while for $\sigma_{+}$and $\sigma_{-}$polarized lasers, the fictitious magnetic fields point opposite.

Therefore we have shown how an effective Hamiltonian like (1) can arise naturally under certain ciucumstances. To get the coefficients $\alpha$ and $\gamma$ determined, it is better to resort to the dipole matrix elements tabulated in Ref. [27].
[1] D. M. Stamper-Kurn, M. R. Andrews, A. P. Chikkatur, S. Inouye, H.-J. Miesner, J. Stenger, and W. Ketterle, Phys. Rev. Lett. 80, 2027 (1998).
[2] T. Ohmi and K. Machida, J. Phys. Soc. Jpn. 67, 1822 (1998); T. L. Ho, Phys. Rev. Lett. 81, 742 (1998).

[3] C. K. Law, H. Pu, and N. P. Bigelow, Phys. Rev. Lett. 
83, 5257 (1998).

[4] M. Koashi and M. Ueda, Phys. Rev. Lett. 84, 1066 (2000).

[5] T. L. Ho and L. Yin, Phys. Rev. Lett. 84, 2302 (2000).

[6] In this paper we assume the total atom number $N$ is even. For $N$ being odd, the AFM ground state is of total spin $S=1$ and thus not strictly isotropic. However, that makes little difference or difficulty to our proposal; see discussions below.

[7] I. B. Mekhov, C. Maschler, and H. Ritsch, Nat. Phys. 3, 319 (2007).

[8] K. D. Bonin and V. V. Kresin, Electric-dipole Polarizabilities of Atoms, Molecules, and Clusters (World Scientific, Singapore, 1997).

[9] F. Brennecke, T. Donner, S. Ritter, T. Bourdel, M. Köhl, and T. Esslinger, Nature (London) 450, 268 (2007); Y. Colombe, T. Steinmetz, G. Dubois, F. Linke, D. Hunger, and J. Reichel, Nature (London) 450, 272 (2007).

[10] In contrast to many experiments (e.g. Refs. [17, 18, 19]), here the $D_{1}$ line is preferred to the $D_{2}$ line. The reason is that under the same detuning, the $D_{1}$-scheme yields the same coupling with the magnetization as the $D_{2}$-scheme, while the coupling with the density is only one half, so the disturbance introduced is smaller.

[11] K. L. Corwin, S. J. M. Kuppens, D. Cho, and C. E. Wieman, Phys. Rev. Lett. 83, 1311 (1999).

[12] J. M. Higbie, L. E. Sadler, S. Inouye, A. P. Chikkatur, S. R. Leslie, K. L. Moore, V. Savalli, and D. M. StamperKurn, Phys. Rev. Lett. 95, 050401 (2005).

[13] Y. Liu, S. Jung, S. E. Maxwell, L. D. Turner, E. Tiesinga, and P. D. Lett, Phys. Rev. Lett. 102, 125301 (2009); Y. Liu, E. Gomez, S. E. Maxwell, L. D. Turner, E. Tiesinga, P. D. Lett, Phys. Rev. Lett. 102, 225301 (2009).

[14] I. Carusotto and E. J. Mueller, J. Phys. B 37, S115 (2004).
[15] J. M. Zhang, W. M. Liu, and D. L. Zhou, Phys. Rev. A 77, 033620 (2008).

[16] J. Kronjäger, C. Becker, P. Navez, K. Bongs, and K. Sengstock, Phys. Rev. Lett. 97, 110404 (2006).

[17] F. Brennecke, S. Ritter, T. Donner, and T. Esslinger, Science 322, 235 (2008); S. Ritter, F. Brennecke, K. Baumann, T. Donner, C. Guerlin, and T. Esslinger, Appl. Phys. B 95, 213 (2009).

[18] S. Gupta, K. L. Moore, K. W. Murch, and D. M. Stamper-Kurn, Phys. Rev. Lett. 99, 213601 (2007).

[19] J. A. Sauer, K. M. Fortier, M. S. Chang, C. D. Hamley, and M. S. Chapman, Phys. Rev. A 69, 051804(R) (2004); K. M. Fortier, S. Y. Kim, M. J. Gibbons, P. Ahmadi, and M. S. Chapman, Phys. Rev. Lett. 98, 233601 (2007).

[20] Experimentally the total atom number $N$ cannot be determined precisely, so we cannot distinguish the AFM and FM ground states just by the shift of the spectra.

[21] J. M. Zhang, W. M. Liu, and D. L. Zhou, Phys. Rev. A 78, 043618 (2008); J. M. Zhang, F. C. Cui, D. L. Zhou, and W. M. Liu, ibid. 79, 033401 (2009).

[22] C. Cohen-Tannoudji and J. Dupont-Roc, Phys. Rev. A 5, 968 (1972).

[23] I. H. Deutsch and P. S. Jessen, Phys. Rev. A 57, 1972 (1998).

[24] D. Cho, J. Korean Phys. Soc. 30, 373 (1997); J. M. Choi and D. Cho, J. Phys. Conf. Ser. 80, 012037 (2007).

[25] The condition that we do not resolve the hyperfine structure of the $D_{1}$ line allows us to take a common denominator.

[26] R. Grimm, M. Weidemüller, and Y. B. Ovchinnikov, Adv. At. Mol. Opt. Phys. 42, 95 (2000).

[27] Daniel A. Steck, "Rubidium 87 D Line Data", available online at http://steck.us/alkalidata (revision 2.0.1, 2 May 2008). 